\title{
SlroliMus Coated angioPlasty Versus Plain Balloon Angioplasty in the tREatment of dialySis acceSs dysfunctION (IMPRESSION): Study Protocol for a Randomized Controlled Trial
}

Suh Chien Pang ( $\nabla$ pang.suh.chien@singhealth.com.sg )

Singapore General Hospital https://orcid.org/0000-0002-9112-0264

Ru Yu Tan

Singapore General Hospital

Edward Choke

Sengkang General Hospital

Jackie Ho

National University of Singapore

Tay Kiang Hiong

Singapore General Hospital

Apoorva Gogna

Singapore General Hospital

Farah Gillan Arani

Singapore General Hospital

Kun Da Zhuang

Singapore General Hospital

Luke Toh

Singapore General Hospital

Shaun Chan

Singapore General Hospital

Pradesh Krishnan

Singapore General Hospital

Kristen Alexa Lee

Singapore General Hospital

Sum Leong

Singapore General Hospital

Richard Lo

Singapore General Hospital

Ankur Patel

Singapore General Hospital 
Bien Soo Tan

Singapore General Hospital

Chow Wei Too

Singapore General Hospital Jasmine Chua

Singapore General Hospital

Alvin Tng

Singapore General Hospital

Tjun Yip Tang

Singapore General Hospital

Siew Ping Chng

Singapore General Hospital

Tze Tec Chong

Singapore General Hospital

\section{Hsien Tsung Tay}

Singapore General Hospital

Hao Yun Yap

Singapore General Hospital

Julian Wong

National University of Singapore

Rajesh Babu Dharmaraj

National University of Singapore

Jun Jie $\mathbf{N g}$

National University of Singapore

Anil Gopinathan

National University of Singapore

Stanley Loh

National University of Singapore

Shao Jin Ong

National University of Singapore

Gary Yoong

National University of Singapore

Jia Sheng Tay

Sengkang General Hospital

Kay Yuan Chong

Singapore General Hospital

Chieh Suai Tan

Singapore General Hospital 


\section{Research Article}

Keywords: Drug-coated balloon, Sirolimus, Dialysis Access Dysfunction, Hemodialysis

Posted Date: August 23rd, 2021

DOl: https://doi.org/10.21203/rs.3.rs-746498/v1

License: (c) (i) This work is licensed under a Creative Commons Attribution 4.0 International License. Read Full License

Version of Record: A version of this preprint was published at Trials on December 1st, 2021. See the published version at https://doi.org/10.1186/s13063-021-05920-3. 
1 SIroliMus Coated angioPlasty Versus Plain Balloon Angioplasty in the tREatment of dialySis acceSs dysfunctION (IMPRESSION): study protocol for a randomized controlled trial

Suh Chien $\mathrm{PANG}^{1 \mathrm{a}}$, Ru Yu TAN ${ }^{1 \mathrm{~b}}$, Edward $\mathrm{CHOKE}^{2 \mathrm{c}}$, Jackie $\mathrm{HO}^{3 \mathrm{~d}}$, Kiang Hiong TAY ${ }^{4 \mathrm{e}}$, Apoorva

4 GOGNA $^{4 \mathrm{f}}$, Farah G IRANI ${ }^{4 \mathrm{~g}}$, Kun Da ZHUANG ${ }^{4 \mathrm{~h}}$, Luke TOH ${ }^{4 \mathrm{i}}$, Shaun CHAN ${ }^{4 \mathrm{j}}$, Pradesh

$5 \quad$ KRISHNAN ${ }^{4 k}$, Kristen A LEE ${ }^{41}$, Sum $L_{E O N G}^{4 m}$, Richard $L^{4 n}$, Ankur PATEL ${ }^{40}$, Bien Soo TAN ${ }^{4 p}$,

6 Chow Wei $\mathrm{TOO}^{4 \mathrm{q}}$, Jasmine $\mathrm{CHUA}^{4 \mathrm{r}}$, Ren Kwang Alvin $\mathrm{TNG}^{1 \mathrm{~s}}$, Tjun Yip TANG ${ }^{5 \mathrm{t}}$, Siew Ping

$7 \mathrm{CHNG}^{5 \mathrm{u}}$, Tze Tec CHONG ${ }^{5 \mathrm{v}}$, Hsien Ts'ung TAY ${ }^{5 \mathrm{w}}$, Hao Yun YAP ${ }^{5 \mathrm{x}}$, Julian WONG ${ }^{3 \mathrm{y}}$, Rajesh Babu

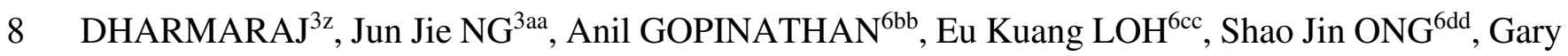

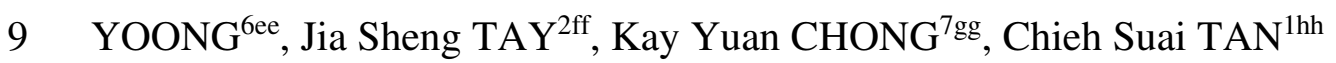

1. Department of Renal Medicine, Singapore General Hospital, Academia, Level 3, 20 College Road, 169856, Singapore

2. Vascular and Endovascular Service, Sengkang General Hospital, 110 Sengkang East Way, 544886, Singapore

3. Department of Cardiac, Thoracic \& Vascular Surgery, National University Hospital, NUHS Tower Block, Level 9, 1E Kent Ridge Road, 119228, Singapore,

4. Department of Vascular and Interventional Radiology, Singapore General Hospital, Outram Road, 169608, Singapore

5. Department of Vascular Surgery, Singapore General Hospital, Academia, Level 5, 20 College Road, Singapore, 169856, Singapore

6. Department of Diagnostic Imaging, National University Health System, 5 Lower Kent Ridge Road, 119074, Singapore

7. Division of Medicine, Singapore General Hospital, SingHealth Tower, Level 5, Singapore

a. pang.suh.chien@singhealth.com.sg

b. tan.ru.yu@ singhealth.com.sg 
c. edward.choke.t.c@singhealth.com.sg

d. surhp@nus.edu.sg

e. tay.kiang.hiong@singhealth.com.sg

f. apoorva.gogna@singhealth.com.sg

g. farah.gillan.irani@singhealth.com.sg

h. zhuang.kun.da@singhealth.com.sg

i. luke.toh.h.w@singhealth.com.sg

j. shaun.xavier.chan@singhealth.com.sg

k. pradesh.kumar.kutty.krishnan@singhealth.com.sg

1. kristen.alexa.lee@singhealth.com.sg

m. leong.sum@singhealth.com.sg

n. richard.lo.h.g@ singhealth.com.sg

o. ankur.patel@singhealth.com.sg

p. tan.bien.soo@singhealth.com.sg

q. too.chow.wei@ singhealth.com.sg

r. jasmine.chua.m.e@singhealth.com.sg

s. alvin.tng.r.k@ singhealth.com.sg

t. tang.tjun.yip@singhealth.com.sg

u. chng.siew.ping@ @inghealth.com.sg

v. chong.tze.tec@singhealth.com.sg

w. tay.hsien.tsung@singhealth.com.sg 
x. yap.hao.yun@singhealth.com.sg

y. julian_wong@nuhs.edu.sg

49

z. rajesh_babu_dharmaraj@nuhs.edu.sg

50

aa.jun_jie_ng@nuhs.edu.sg

51 bb. anil_gopinathan@nuhs.edu.sg

52

cc. stanley_loh@nuhs.edu.sg

53

dd.shao_jin_ONG@nuhs.edu.sg

54

ee. sem_yoong@nuhs.edu.sg

55

ff. tay.jia.sheng@singhealth.com.sg

56

gg. chong.kay.yuan@sgh.com.sg

57

hh.tan.chieh.suai@singhealth.com.sg 


\section{Abstract}

Background: Percutaneous transluminal angioplasty is the current standard treatment for arteriovenous fistula (AVF) stenosis. The mid- and long-term patency with plain old balloon angioplasty (POBA) is however far from satisfactory. While paclitaxel-coated balloon angioplasty has been shown to be superior to POBA, concern over its safety profile has recently arisen after reported possible increased mortality risk with a meta-analysis of large lower limb studies. An angioplasty balloon with a new type of drug coating, the sirolimus coated balloon (SCB) has been proven to improve patency in the coronary arteries. However, its effect on AV access has yet to be studied.

Methods/Design: This is an investigator-initiated, prospective, multicenter, doubled blinded, randomized controlled clinical trial to assess the effectiveness of SCB compared to POBA in improving the patency of AVF after angioplasty. A total of 170 patients with mature AVF that requires PTA due to AVF dysfunction will be randomly assigned to treatment with a SCB or POBA at a 1:1 ratio, stratified by location of AVF and followed-up for up to 1 year. Inclusion criteria includes (1) adult patient aged 21 to 85 years who requires balloon angioplasty for dysfunctional arteriovenous fistula, (2) matured AVF, defined as being in use for at least 1 month prior to the angioplasty, (3) successful angioplasty of the underlying stenosis with POBA, defined as less than 30\% residual stenosis on Digital Subtraction Angiography (DSA) and restoration of thrill in the AVF on clinical examination. Exclusion criteria include thrombosed or partially thrombosed access circuit at the time of treatment, presence of symptomatic or angiographically significant central vein stenosis that require treatment with more than $30 \%$ residual stenosis post angioplasty, and existing stent placement within the AVF circuit. The primary end point of the study is access circuit primary patency at 6 months. The secondary end points are target lesion primary patency, circuit assisted primary patency, circuit secondary patency at 3,6 and 12 months, target lesion restenosis rate at 6 months, total number of interventions, complication rate and cost 
83 effectiveness. The trial is supported by Concept Medical.

84 Discussion: This study will evaluate the clinical efficacy and safety of SCB compared to 85 POBA in the treatment of AVF stenosis in hemodialysis patients.

86

87 Trial registration: ClinicalTrials.gov Identifier: NCT04409912 on 1 June 2020

88 https://clinicaltrials.gov/ct2/show/NCT04409912

89 Protocol Version: 4

\section{Keywords}

91 Drug-coated balloon, Sirolimus, Dialysis Access Dysfunction, Hemodialysis

92

93

94

95

96

97

98

99

100

101

102

103

104

105

106

107 


\section{Background}

Despite significant advances in medical technologies, arteriovenous fistula (AVF) dysfunction remains a major morbidity for patients with end stage renal disease (ESRD) who are dependent on hemodialysis.(1) Neointimal hyperplasia (NIH) contributed by endothelial injury from shear stress and turbulent blood flow frequently results in clinically significant stenosis, leading to diminished blood flow and thrombosis in some accesses.(2, 3) Percutaneous transluminal angioplasty (PTA) is the current therapy of choice for AVF dysfunction. However, AVF patency rates post-PTA are often hampered by endothelial denudation and further NIH caused by mechanical dilatation of stenosis with angioplasty balloons. (3) With significant scientific advances in understanding the mechanism of AVF stenosis, medical technology innovations to improve patient care and AVF outcomes have been emerging.

Drug-coated balloon (DCB) devices are one of the most exciting technologies available in recent years. By inhibiting the proliferative response to the acute trauma caused by balloon angioplasty, the DCB has shown its efficacy with improved primary patency rates of AVF posttreatment. To date, several randomized controlled trials have shown the superiority of paclitaxelcoated balloon (PCB) angioplasty over conventional plain old balloon angioplasty (POBA) in the treatment of AV access stenosis (4-8). However, safety concerns had also arisen recently after a metaanalysis of lower limb studies reported higher 12-month mortality of 7.6\% vs. $5.8 \%$ for paclitaxelcoated devices compared to uncoated devices. (9) Although similar risks have not been demonstrated in meta-analysis on ESRD patients receiving PCB for AVF intervention(10), the safety concern on PCB invariably may limit its use, which may affect the patency rates of AV accesses of ESRD patients. On the other hand, the use of stent-graft in hemodialysis access has shown improved patency rates in arteriovenous graft $(\mathrm{AVG})(11-14)$, but its role in AVF has been controversial as it may reduce the length of vessel available for cannulation. 
133 alternative to PCB. Clinical studies in coronary artery intervention using SCB for in-stent stenosis 134 and small vessel disease have shown excellent procedural success and 6-month post procedural 135 patency $(15,16)$. Compared to paclitaxel, sirolimus is cytostatic and has a wide therapeutic index 136 indicating a more favorable safety profile. In addition, sirolimus has been used in ESRD patients who 137 have received renal transplantation as immunosuppressive agents for decades at a much higher dosage 138 than the dose on SCB.

\section{Trial objectives}

The IMPRESSION study aims to examine the effectiveness and safety of SCB angioplasty compared with POBA in the treatment of AVF stenosis.

\section{Trial design}

This multicenter, prospective, parallel, double-blinded, randomized controlled trial is an investigator-initiated study. The patients are randomized at a 1:1 ratio to receive either SCB (intervention arm) or POBA (placebo arm) following successful angioplasty of AVF stenosis. Randomization will be stratified by location of AVF (above vs below elbow) to ensure an even distribution of AVF by location between both groups.

\section{Methods: Participants, interventions, and outcomes}

\section{$\underline{\text { Participants }}$}

The study will recruit a total of 170 patients from 3 different hospitals in Singapore over four 153 years. The potential participants are patients who are scheduled for PTA of dysfunctional AVF by their primary physician. The potential participants will be screened for eligibility according to the inclusion and exclusion criteria for the study summarized in table 1 by study team member. Eligible patients are offered enrolment. As one of the eligibility criteria (residual stenosis $<30 \%$ ) can only be 
determined during the procedure, informed consent is taken from eligible participants before the procedure. The study team members will obtain written informed consent to participate in the study from all enrolled participants. Additional informed consent for the collection and the use of participant data will also obtained. An enrolment number is given to each participant for the purpose 161 of anonymization.

$\underline{\text { Interventions }}$

After enrolment, the patient will undergo PTA procedures in the study sites' interventional suites equipped with a fluoroscopy machine with the ability to perform digital subtraction angiography and post-processing software for quantitative vascular analysis. Fistulograms of the entire dialysis circuit from the feeding artery, arteriovenous anastomosis to the central veins will be performed. The target lesions will be treated in the standard fashion with POBA. When there is more than one stenosis, all the lesions will be labeled and treated accordingly. Lesions are considered separate if they are separated by a gap of at least $2 \mathrm{~cm}$. The operator will angioplasty each lesion with a POBA sizing that is identical to the adjacent reference vessel. Inflation time will be at least 1 minute

171 per inflation. If there is significant residual stenosis after initial angioplasty, repeat angioplasty with 172 a larger diameter, high-pressure, or cutting balloon may be used at the operator's discretion. In the stenotic segment adjacent to an aneurysmal segment, where the percentage of stenosis is difficult to determine, vessel diameter must reach at least $6 \mathrm{~mm}$ to be considered for inclusion.

\section{$\underline{\text { Randomization procedures and study devices }}$}

Once all the target lesions are adequately treated, as visually assessed by the operator to have less than $30 \%$ residual stenosis, the patients will be randomized, stratified to the site of AVF (above 178 or below elbow) with a secure web-based randomization program developed by the Singapore

179 Clinical Research Institute. Treatment allocation generated by the program will be available only 180 upon request from the operator. The diameter of the study balloon chosen should be similar to the 181 size of the adjacent healthy segment of the blood vessel, and balloon length selection will be at the 
operator's discretion. The study balloons are inflated to the appropriate pressure, not exceeding the rated burst pressure, for at least 2 minutes. Completion venograms of all the target lesions are performed following treatment with study balloons.

The study balloons (sirolimus coated and placebo balloon) used in both arms are custom-made by the same manufacturer (Concept Medical Research Private Limited, India), have the same profile, inflation pressure, and identical packaging. In addition, the balloons are labelled as " $\mathrm{A}$ " and " $\mathrm{B}$ " to maintain the blinding. All the balloons are of the $0.035 "$ platform and available in diameters of 5, 6 , 7, $8,12 \mathrm{~mm}$, lengths of $60,80,100 \mathrm{~mm}$, and shaft length of 45 or $90 \mathrm{~cm}$. The dosing of sirolimus on $\mathrm{SCB}$ is $1.25 \mu \mathrm{g} / \mathrm{mm}^{2}$.

\section{$\underline{\text { Blinding }}$}

The assigned operator performing the procedure will be different from the study member who performed treatment allocation. The participants, referring physicians, investigators assessing postintervention outcomes, dialysis center staff, investigators performing follow-up, the operator performing the PTA and the data analysis team will be blinded to treatment allocation. During the procedure, the participant will not know which treatment they will be receiving as they will not be able to see the procedure with their views completely blocked from the operative field by sterile drapes. The operator will be instructed of which balloons ('A' or 'B') to use but they will not know whether these balloons are sirolimus coated balloons or the placebo. Only designated study coordinators handling the investigational product will know the treatment allocation as it is not feasible for them to administer the trial blinded.

\section{$\underline{\text { Unblinding procedure }}$}

Unblinding will occur at the end of the study (the 12 month follow up of the last recruited participant). At this stage, all blinded research data would have been collected and unblinding will allow the data analyses to occur. In case of an emergency requiring information on treatment allocation for patient management, the principal investigator will break the blind for the patient. 
Patients with resistant stenosis that cannot be successfully treated with balloon angioplasty,

209 has partial thrombosis of the AVF or require stent deployment will not be eligible for randomization.

210 Such cases are considered screen failures and will be replaced. These participants will still receive 211 the standard care with POBA.

212 The participants are free to withdraw their consent and discontinue participation at any time 213 during the trial without prejudice to their medical care. The data that has been collected until the time 214 of withdrawal will be kept and analyzed to enable comprehensive evaluation and maintain the 215 scientific validity of this study. In the event of voluntary withdrawal, the participant will be asked for 216 permission to continue clinic follow-up for assessment of safety outcomes. Any adverse events will 217 be monitored and treated till resolution. Participants who choose to withdraw will not be replaced. As 218 ESRD patients have a risk of sudden death, if any study participant passes away during their study 219 participation, they will automatically be considered as study withdrawals and replaced.

\section{$220 \quad$ Post-procedure assessment and follow-up}

$221 \quad$ Immediate assessment

As ultrasound is used as an imaging tool to monitor the AVF post intervention for the study, ultrasound assessment of the AVFs will be performed by a trained operator to document the diameter of the vessels within 24 hours post PTA. Volume flow rates at the mid-brachial artery and venous outflow will be recorded. The minimum diameter of each target lesion will be recorded.

3-, 6- and 12-month assessment

All participants will be followed up for up to 1 year. The window periods for the post-PTA visits are 3-month \pm 1 week, 6-month \pm 4 weeks and 12-month \pm 4 weeks. During the follow-up period, ultrasound assessment will be performed in each study site's vascular study unit. Reminder will be given to participants by study coordinator to ensure adherence to follow up ultrasound. The 
brachial artery and venous outflow, minimum diameter of each target lesion, and any new stenosis

233 within the AVF circuit. For patients who may have undergone or are planned for fistulograms, the 234 fistulograms may be used instead of the scheduled ultrasound. A study team member who is not 235 involved in the index procedure will be responsible for reviewing the patient, ultrasound images or 236 reports, and hemodialysis charts during each follow-up and determine plans for repeat intervention 237 when clinically indicated.

238 Contingency plan

239 In situations where hospital visits are limited to essential visits only, patient will not be able 240 to return for follow-up ultrasound scans. The study team will review the patient's medical record, 241 dialysis records from patient's dialysis centers and conduct telephone consult with the patient to 242 collect data in place of the ultrasound scan.

243 Repeat intervention on AVF

244 Repeat intervention will be performed when a decrease in access flow is associated with 245 clinically significant lesions as recommended by the National Kidney Foundation's Kidney Disease 246 Outcomes Quality Initiative (KDOQI) clinical practice guideline for vascular access: 2019 update. 247 (Table 2) (17) Patients who require repeat intervention on the AVF are considered to have reached 248 the primary endpoint.

249 Central laboratory assessment

250 The index procedural fistulograms and all follow-up ultrasound images will be sent to a 251 central laboratory for review by a group of independent assessors. The independent assessors will use 252 quantitative vascular analysis software (Syngo, Siemens Healthcare, Erlangen, Germany) as an 253 adjunct to evaluate the lesions from fluoroscopy images in the picture archiving and communication 254 system (PACS). The central laboratory's interpretation of all angiograms will be used for the data 255 analyses. 


\section{Outcomes}

In this study, standard definitions for patency outcomes, major and minor complication rates according to the Society of Interventional Radiologist (SIR) guidelines are used. (18)

260 Primary endpoints

261 Efficacy endpoint

Safety endpoint

1. Complication rates at 1-,3-, 6- and 12-month according to Society of Interventional Radiology (SIR) definitions of minor or major complications. (18)

$\underline{\text { Secondary endpoints }}$

1. Time taken to the next intervention

2. Treated lesion percent stenosis at 6- and 12-months with ultrasound

3. Treated lesion restenosis rate at 6-months

4. Number of repeat interventions to treated lesion at 6- and 12-months

5. Number of repeat interventions to maintain access circuit at 6- and 12-months (including interventions to treated lesion)
6. Treated lesion revascularization free interval

7. De novo stenosis detected on ultrasound scan at 3-, 6- and 12-months

8. Post intervention treated lesion patency at 3-, 6- and 12-months

9. Post-intervention primary patency at 3-, 6- and 12-months

10. Post-intervention assisted primary patency at 3-, 6- and 12-months

11. Post-intervention secondary patency at 3-, 6- and 12-months

Circuit primary patency rates is defined as the percentage of patients whose AVF remain patent 281 hospitalization < 40 hours, requiring major therapy, unplanned increase in level of care, prolonged 
hospitalization ( $>48$ hours), leading to permanent adverse sequelae, or death. A minor complication requires no therapy with no consequences or requires nominal therapy with no consequences, including overnight admission for observation only. (18)

Time taken to the next intervention is defined as the number of months from index angioplasty to the subsequent intervention or till study completion at 12 month. Treated lesion percent stenosis is defined as percent stenosis relative to adjacent reference vessel, [1 - (minimum lesion diameter / reference vessel diameter)] x 100) on 6- and 12-months follow-up ultrasound. Restenosis rate is defined as the incidence of more than $50 \%$ diameter narrowing of the target lesion compared to adjacent vessel segment at 6-month follow-up ultrasound scan. Treated lesion re-intervention free interval is defined as the interval from index angioplasty to repeat clinically driven target lesion intervention, anytime within the 12-months study period.

Post-intervention primary patency is defined as the percentage of patients whose AVF remains patent and does not require any further interventions (18), while post-intervention treated lesion patency is measured as the percentage of patients whose AVF remains patent and does not require any additional interventions at 3-, 6-, and 12-months after the index angioplasty. These outcomes are determined by ultrasound imaging or angiogram or clinical examination. The decision for reintervention based on clinical examination findings include loss of thrill, pulsatile flow, or swollen arm. Post-intervention assisted primary patency is defined as the percentage of patients whose AVF requires additional interventions to remain patent and post-intervention secondary patency is measured as the percentage of patients whose AVF have thrombosed and require additional procedure determined by clinical history during the study period. 
We assume that the SCB will have similar effectiveness as the PCB based on our pilot study

308 (6-month target lesion primary patency of 82.9\% with SCB) and previous meta-analysis (pooled 6-

309 month target lesion primary patency $73.7 \%$ with PCB). Considering a dropout rate of $10 \%$, a

310 sample size of 170 patients randomized into a 1:1 ratio will have $80 \%$ power to detect a difference

311 between the two groups at 6-month.

\section{Primary Outcome}

Both the primary efficacy outcome and primary safety outcome will be analyzed using an intention to treat (ITT) analysis set which includes all randomized subjects. The ITT subjects will be analyzed according to their randomized group assignment irrespective of the treatment delivered and subject follow-up time, and all events post- randomization will be counted toward study endpoints. The count and percentage of subjects with each outcome will be presented by treatment. The percentage of the efficacy endpoint will be based on the subjects who had non-patency event (i.e., CD-TLR or access circuit thrombosis) within 210 days post procedure or had no non-patency event but followed up for at least 150 days. The efficacy endpoint will be compared between treatments using the Z-test (Z-test approximation to a binomial distribution) as the primary analysis method. The percentage of the primary safety endpoint will be based on subjects who had AV-access-circuitrelated-SAE within 30 days post-procedure or had no AV-access-circuit-related-SAEs but were followed up for at least 23 days. Non-inferiority on the safety endpoint will be tested using the Farrington- Manning method. The differences between treatments and the corresponding 95\% confidence interval (CI) will be calculated. To control the overall Type I error the following sequential analysis approach will be taken:

- Primary efficacy superiority; if significant at one-sided alpha=0.025, and

- Primary safety non-inferiority; if significant at one-sided alpha=0.025, then proceed to key secondary endpoints 
331 The study will be deemed a success if both the superiority of efficacy and non-inferiority of safety

332 are demonstrated. Additional analysis of the primary endpoint using time to the events will be 333 evaluated according to Kaplan-Meier method, and the log-rank tests will be applied to compare the 334 survival curves over time between the treatments for each primary endpoint respectively.

\section{Secondary Outcome}

Descriptive statistics for the secondary endpoints will be provided. Unless otherwise specified, for categorical variables, the count and percentage of subjects with each outcome will be presented. They will be evaluated by using chi-square tests or Fisher's exact tests depending on the event counts. Continuous variables will be compared with t-tests. The differences between treatments together with the corresponding $95 \%$ confidence interval will be calculated. Additional time to event survival analysis will be employed when applicable. Secondary endpoints will be analyzed using ITT analysis set, per- protocol analysis set, and as treated analysis set respectively when applicable. The key secondary endpoints will be compared between treatments sequentially by using ITT analysis set in a superiority manner if the two primary endpoint tests pass, each at a one-sided significance level of 0.025 .

As treated analysis set include randomized subjects who received a SCB or PBA. The as treated subjects will be analyzed according to the device subjects received. If the as treated analysis set is different from ITT analysis set, the primary and secondary endpoints will be analyzed on as treated analysis set to assess the sensitivity. Per-Protocol Analysis set include subjects who have: (a) received the randomized treatment as assigned without provisional stenting or other potential bailout

351 procedure; (b) no pre-specified inclusion/exclusion violation(s); and (c) available endpoint data post352 index procedure. Per-Protocol Analysis set will be applied to primary and key secondary endpoint 353 analyses.

\section{$354 \quad$ Safety Analysis}


All Adverse events (AEs) post informed consent will be collected and presented in a listing.

The AEs started during or post index procedure through the end of study will be tabulated. The AEs,

Significant adverse events (SAEs), and AEs leading to death, will be summarized by treatment, system organ class and by time. The relationship of AEs to procedure, device and therapy will also be summarized respectively. Fisher exact test will be used to test treatment difference.

\section{Ethics and regulatory approvals}

The trial will be conducted in compliance with the principles of the Declaration of Helsinki

(1996). This study protocol and all its related documents have been approved by local Institutional

Review Board (reference: 2019/2896). Informed consent will be obtained from all subjects

participating in the study. In the event the patient is unable to write, informed consent can be given via a thumbprint or orally in the presence of at least one witness in accordance with the Medical Research Involving Human Subjects Act (article 6, subsection 2, altered WMO).

\section{Data handling \& Auditing}

Hardcopy source documents will be used to collect required data. The contained information will then be entered into the Electronic Research Data Capture (REDCap) system by study coordinator. The hardcopy source document and list containing the links between enrolment number of each participant to their identities will be kept under lock and key cabinets. Online database / electronic case report forms will be password protected. Only the principal investigator, designated co-investigators and study coordinators will have access to the research data. Access to the hardcopy data will be controlled. Access to the electronic database / case report forms will be password protected and login recorded. The images from the angiogram and intervention and ultrasound scans are recorded into the electronic picture archiving and communication system (PACS) of a password protected computer as per routine clinical practice in the study site. These records will be anonymized and saved in a hard disk for review by independent assessors. 
The data and safety monitoring will be performed by the principal investigator and a team of co-investigators. The principal investigator and study coordinators will be responsible for the dissemination of data and safety information to the study sites. This will be communicated via faceto-face meetings and emails using secure institution password protected electronic communications.

\section{Assessment of Safety}

All adverse events are recorded in the case report forms. The principal investigator and coinvestigator will monitor safety data by reviewing the case report forms. All serious adverse events will be notified by the principal investigator to the CIRB within the stipulated timeframe. Follow-up information will be actively sought and submitted as it becomes available.

\section{Discussions}

We present the protocol of a multi-center RCT to evaluate the efficacy of SCB in improving patency rates of AVFs from reduction in restenosis and reintervention rates. SCBs have been used successfully in hemodialysis accesses in small, non-randomized studies. Tan et al. reported 3- and 6month primary patency of $76 \%$ and $65 \%$ with application of SCB at the graft vein junction after successful thrombectomy of AVG (19), while Tang et al. reported 3- and 6-month target lesion primary patency of $97.9 \%$ and $82.9 \%$ following treatment with SCB for dysfunctional AVF. (20) Although both studies suggested that SCB may be safe and efficacious for treatment of hemodialysis access dysfunction, one cannot draw a conclusion from these small, pilot studies without control groups.

To the author's knowledge, this is the first RCT comparing SCB use versus POBA in 401 dysfunctional AVF. Double blinding is used in the study to minimize potential bias. To reduce confounding effect from non-maturing AVF that may have a different pathogenesis, the study 403 includes only mature AVF which has been in use for at least 1 month. In addition, randomization is 
groups, as above-elbow AVFs generally have larger vessel size and may have better outcomes than below-elbow AVFs.

Circuit primary patency instead of target lesion primary patency is chosen as the primary endpoint because the study is designed to treat all stenosis in the AVF circuit. Furthermore, it is the 409 circuit patency that is most meaningful to the patients with numerous economical and psychosocial 410 benefits from a lesser need for reintervention. In accordance with the KDOQI Clinical Practice 411 Guideline for Vascular Access 2019, reintervention of AVF is based on clinical indications as summarized in Table 2. (17). A recruited patient who has concurrent asymptomatic central vein stenosis will not receive intervention for the central vein stenosis as previous evidence showed a lack of benefit in treatment in this group of patients. (21)

We learned from previous DCB studies $(4,5,7,8,22)$ that adequate vessel preparation with high-pressure angioplasty balloons is a crucial component of PTA. Although these studies were designed differently, they showed similar primary efficacy endpoint of target lesion at 6-month with DCB after appropriate vessel preparation, likely because adequate pretreatment enhances drug penetration into the vessel wall for maximal pharmaceutical effects to inhibit NIH.

In this study, we use ultrasound as a tool to assess the AVF following angioplasty, this is to examine and document the degree of post-procedural elastic recoil within 24 hours. This will help determine whether the stenosis seen in subsequent follow-up ultrasound is part of the NIH process or recoil post-angioplasty. The rate of late lumen loss may also be compared accurately between the 2 groups. Ultrasound assessment also allows a detailed evaluation of any potential vascular injury/dissection during the index procedure and monitor for recovery. Systemic use of sirolimus has been associated with an increased risk of impaired wound healing. (23) Locally delivered sirolimus was thought to be responsible for impaired re-endothelialization and lead to aneurysm formation following sirolimus-eluting stent placement reported in the coronary arteries interventions. $(24,25)$ 
to be detected, recorded, and followed up longitudinally. We anticipate that the result of this trial will

431 provide additional insight into the effort to improve patency outcomes in AVF for ESRD patients.

\section{Trial status}

433 Recruitment started on January 11, 2021. The projected timeline for recruitment and follow up is 434 expected to finish by May 2024.

\section{List of abbreviations:}

436 AVF - Arteriovenous fistula

437 NIH - Neointimal hyperplasia

438 DCB - Drug-coated balloon

439 SCB - Sirolimus-coated balloon

$440 \quad$ AV - Arteriovenous

441 PCB - Paclitaxel-coated balloon

442 ESRD - end stage renal disease

443 DSA - Digital Subtraction Angiography

444 PTA - Percutaneous transluminal angioplasty

\section{Declarations}

447 Protocol amendment: Amendments are submitted to and approved by our institution's CIRB. 448 Version 2 dated on 23 Dec 2020; inclusion criteria of residual stenosis changed from $<25 \%$ to $<30 \%$ 449 as that is the standard clinical level. Version 3 dated on 09 Feb 2021; follow-up ultrasound scans are 450 to be conducted at each site instead of a primary site as the study is carried out during the COVID 45119 pandemic when movement restriction is in place. Version 4 dated on 24 May 2021; addition of 452 inclusion criteria for patient with concurrent asymptomatic or angiographically not significant central 453 vein stenosis if no treatment during the index procedure is required. Version 4 also includes addition 454 to indications of clinically significant lesions for repeat intervention based on the latest 2019 KDOQI 
guideline which was published after the initial protocol was written. The amendments did not change the original objectives and/or alignment of the study.

457 Consent for publications: Manuscripts will be sent to the institutional representative prior to 458 submission. All study members with significant roles in the trial will be acknowledged.

459 Availability of data and material: The datasets generated and/or analyzed during the current study 460 are not publicly available due to confidentiality of the data but are available from the corresponding 461 author on reasonable request.

462 Competing interests: The authors declare that they have no competing interests.

463 Funding: This work was funded and supported by Concept Medical who have no role in the design, 464 running, or analysis of the trial. All study angioplasty balloons are supplied and the cost of travel for 465 study visit, follow up ultrasound scan, hiring of study coordinators and biostatistician services are 466 covered by the sponsor.

467 Authors' contributions: CST is the principal investigator of the study. EC, JH are the lead site 468 investigator from two other hospital. SCP, RYT, KHT, AG, FGA, KDZ, LT, SC, PK, KAL, SL, RL, 469 AP, BST, CWT, JC, RKAT, TYT, SPC, TTC, HTT, HYY, JW, RBD, JJN, AG, EKL, SJO, GY, JST 470 are co-investigators. KYC is the lead study coordinator. All contributed to the study design and 471 reviewed the paper. CST, SCP, RYT, RKAT and KYC read and approved the final manuscript.

Acknowledgement: The authors wish to thank Sidhu Dalpreet KAUR, Pei Ling CHEAH, Nur Nabila Binte TAUFIQ, Lingyan MENG, Wan Ling TAN, Eilane Yi Ling PEH for their dedication to coordinate for this study. 
481 1. Campos RP, Do Nascimento MM, Chula DC, Do Nascimento DE, Riella MC. Stenosis in 482 hemodialysis arteriovenous fistula: evaluation and treatment. Hemodial Int. 2006;10(2):152-61. 483 2. Roy-Chaudhury P, Arend L, Zhang J, Krishnamoorthy M, Wang Y, Banerjee R, et al. 484 Neointimal hyperplasia in early arteriovenous fistula failure. Am J Kidney Dis. 2007;50(5):782-90. 485 3. Roy-Chaudhury P, Sukhatme VP, Cheung AK. Hemodialysis vascular access dysfunction: a 486 cellular and molecular viewpoint. Journal of the American Society of Nephrology: JASN. $487 \quad 2006 ; 17(4): 1112-27$.

488 4. Katsanos K, Karnabatidis D, Kitrou P, Spiliopoulos S, Christeas N, Siablis D. Paclitaxel489 coated balloon angioplasty vs. plain balloon dilation for the treatment of failing dialysis access: 6490 month interim results from a prospective randomized controlled trial. Journal of endovascular 491 therapy : an official journal of the International Society of Endovascular Specialists. $492 \quad 2012 ; 19(2): 263-72$.

493 5. Kitrou PM, Spiliopoulos S, Katsanos K, Papachristou E, Siablis D, Karnabatidis D.

494 Paclitaxel-coated versus plain balloon angioplasty for dysfunctional arteriovenous fistulae: one-year 495 results of a prospective randomized controlled trial. Journal of vascular and interventional radiology 496 : JVIR. 2015;26(3):348-54.

497 6. Lookstein RA, Haruguchi H, Ouriel K, Weinberg I, Lei L, Cihlar S, et al. Drug-Coated 498 Balloons for Dysfunctional Dialysis Arteriovenous Fistulas. The New England journal of medicine. $499 \quad 2020 ; 383(8): 733-42$.

500 7. Swinnen JJ, Hitos K, Kairaitis L, Gruenewald S, Larcos G, Farlow D, et al. Multicentre, 501 randomised, blinded, control trial of drug-eluting balloon vs Sham in recurrent native dialysis 502 fistula stenoses. The journal of vascular access. 2019;20(3):260-9. 
503 8. Trerotola SO, Lawson J, Roy-Chaudhury P, Saad TF. Drug Coated Balloon Angioplasty in 504 Failing AV Fistulas: A Randomized Controlled Trial. Clinical journal of the American Society of 505 Nephrology : CJASN. 2018;13(8):1215-24.

506 9. Katsanos K, Spiliopoulos S, Kitrou P, Krokidis M, Karnabatidis D. Risk of Death Following 507 Application of Paclitaxel-Coated Balloons and Stents in the Femoropopliteal Artery of the Leg: A 508 Systematic Review and Meta-Analysis of Randomized Controlled Trials. Journal of the American 509 Heart Association. 2018;7(24):e011245.

510 10. Kennedy SA, Mafeld S, Baerlocher MO, Jaberi A, Rajan DK. Drug-Coated Balloon 511 Angioplasty in Hemodialysis Circuits: A Systematic Review and Meta-Analysis. Journal of 512 vascular and interventional radiology : JVIR. 2019;30(4):483-94.e1.

513 11. Haskal ZJ, Saad TF, Hoggard JG, Cooper RI, Lipkowitz GS, Gerges A, et al. Prospective, 514 Randomized, Concurrently-Controlled Study of a Stent Graft versus Balloon Angioplasty for 515 Treatment of Arteriovenous Access Graft Stenosis: 2-Year Results of the RENOVA Study. Journal 516 of vascular and interventional radiology : JVIR. 2016;27(8):1105-14.e3.

517 12. Haskal ZJ, Trerotola S, Dolmatch B, Schuman E, Altman S, Mietling S, et al. Stent graft 518 versus balloon angioplasty for failing dialysis-access grafts. The New England journal of medicine. $519 \quad 2010 ; 362(6): 494-503$.

520 13. Kouvelos GN, Spanos K, Antoniou GA, Vassilopoulos I, Karathanos C, Matsagkas MI, et 521 al. Balloon Angioplasty Versus Stenting for the Treatment of Failing Arteriovenous Grafts: A 522 Meta-Analysis. European journal of vascular and endovascular surgery : the official journal of the 523 European Society for Vascular Surgery. 2018;55(2):249-56.

524 14. Vesely T, DaVanzo W, Behrend T, Dwyer A, Aruny J. Balloon angioplasty versus Viabahn 525 stent graft for treatment of failing or thrombosed prosthetic hemodialysis grafts. Journal of vascular 526 surgery. 2016;64(5):1400-10.e1. 
15. Jim MH, Fung RC, Yiu KH. Angiographic result of sirolimus-eluting balloon in de novo small coronary artery lesion (ARSENAL). International journal of cardiology. 2016;222:992-4.

530 (Sirolimus Angioplasty Balloon for Coronary In-Stent Restenosis): Angiographic Results and 1-

531 Year Clinical Outcomes. JACC Cardiovascular interventions. 2017;10(20):2029-37.

532 17. Lok CE, Huber TS, Lee T, Shenoy S, Yevzlin AS, Abreo K, et al. KDOQI Clinical Practice 533 Guideline for Vascular Access: 2019 Update. American journal of kidney diseases : the official 534 journal of the National Kidney Foundation. 2020;75(4 Suppl 2):S1-s164.

535 18. Gray RJ, Sacks D, Martin LG, Trerotola SO. Reporting standards for percutaneous 536 interventions in dialysis access. J Vasc Interv Radiol. 2003;14(9 Pt 2):S433-42.

537 19. Tan CW, Tan RY, Pang SC, Tng ARK, Tang TY, Zhuang KD, et al. Single-Center 538 Prospective Pilot Study of Sirolimus Drug-Coated Balloon Angioplasty in Maintaining the Patency 539 of Thrombosed Arteriovenous Graft. J Vasc Interv Radiol. 2021;32(3):369-75.

540 20. Tang TY, Soon SXY, Yap CJQ, Chan SL, Tan RY, Pang SC, et al. Early (6 months) results 541 of a pilot prospective study to investigate the efficacy and safety of sirolimus coated balloon 542 angioplasty for dysfunctional arterio-venous fistulas: MAgicTouch Intervention Leap for Dialysis 543 Access (MATILDA) Trial. PLoS One. 2020;15(10):e0241321.

544 21. Renaud CJ, Francois M, Nony A, Fodil-Cherif M, Turmel-Rodrigues L. Comparative 545 outcomes of treated symptomatic versus non-treated asymptomatic high-grade central vein stenoses 546 in the outflow of predominantly dialysis fistulas. Nephrol Dial Transplant. 2012;27(4):1631-8. 547 22. Irani FG, Teo TKB, Tay KH, Yin WH, Win HH, Gogna A, et al. Hemodialysis 548 Arteriovenous Fistula and Graft Stenoses: Randomized Trial Comparing Drug-eluting Balloon 549 Angioplasty with Conventional Angioplasty. Radiology. 2018;289(1):238-47. 
550 23. Valente JF, Hricik D, Weigel K, Seaman D, Knauss T, Siegel CT, et al. Comparison of 551 Sirolimus vs. Mycophenolate Mofetil on Surgical Complications and Wound Healing in Adult 552 Kidney Transplantation. American Journal of Transplantation. 2003;3(9):1128-34.

553 24. Hofma SH, van der Giessen WJ, van Dalen BM, Lemos PA, McFadden EP, Sianos G, et al. 554 Indication of long-term endothelial dysfunction after sirolimus-eluting stent implantation. European 555 heart journal. 2006;27(2):166-70.

556 25. Zbinden R, Eshtehardi P, Cook S. Coronary aneurysm formation in a patient early after 557 everolimus-eluting stent implantation. The Journal of invasive cardiology. 2008;20(5):E174-5. 558 


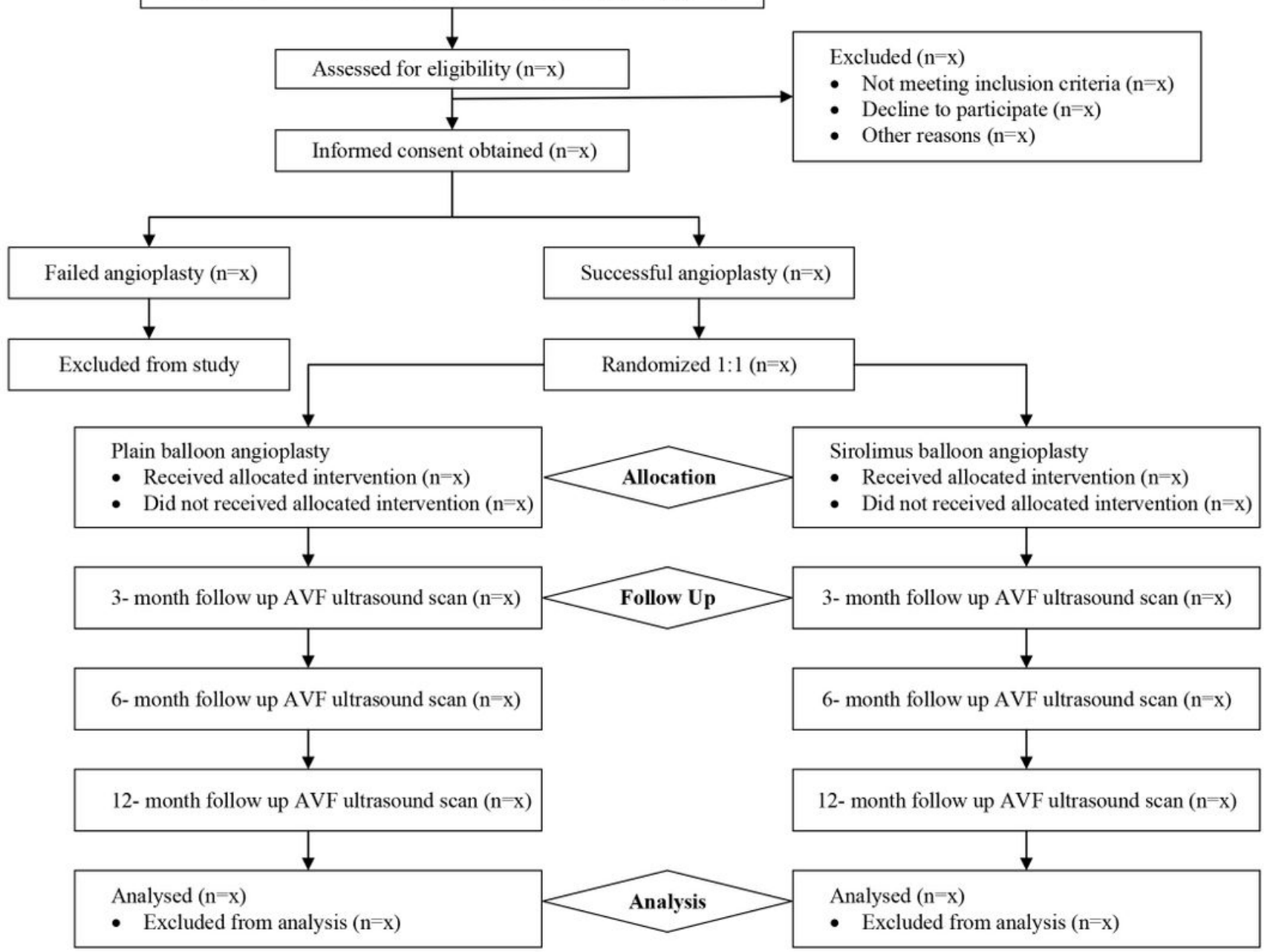

Figure 1

Flowchart of study based on the Consolidated Standards for Reporting of Trials

\section{Supplementary Files}

This is a list of supplementary files associated with this preprint. Click to download.

- ImpressionStudyProtocoltable.pdf

- Adverseeventform.pdf

- ConsentFormHospital1.pdf

- ConsentFormHospital2.pdf 
- ConsentFormHospital3.pdf

- NoScancontingencyForm.pdf

- OutcomesSummaryForm.pdf

- PostlnterventionScanForm.pdf

- ScanFormat12months.pdf

- ScanFormat3months.pdf

- ScanFormat6months.pdf

- ScreeningForm.pdf

- TreatmentForm.pdf

- UnscheduledVisitForm.pdf 